\title{
LINE FORMATION IN U GEM AND T LEO
}

W. HUMMEL ${ }^{1}$, K. HORNE ${ }^{2}$, T. R. MARSH ${ }^{3}$, JANET H. WOOD ${ }^{4}$ 1. Astrofysisch Instituut, Vrije Universiteit Brussel, Pleinlaan 2, B-1050 Brussel, Belgium

2. University of St. Andrews, School of Physics \&3 Astronomy, North Haugh, St. Andrews, Fife KY16 9SS, Scotland

3. University of Southampton, Department of Physics, Highfield, Southampton SO17 1BJ, England

4. Keele University, Department of Physics, Keele ST5 5BG, England

We present 3-D LTE radiative transfer calculations [1] for $\mathrm{H}, \mathrm{He}$ and $\mathrm{Ca}$ in accretion disks (AD) of dwarf novae in quiescence. The model disk is assumed to be in hydrostatic equilibrium vertically, and to rotate with Keplerian velocities. Calculated emission lines are fitted to phase-averaged, continuum-subtracted spectra of U Gem (Fig. 1) and T Leo (Fig. 2). Up to four parameters of the $\mathrm{AD}$ have been fitted: distance $D$, baryonic number density $N$, isotropic turbulence $V_{\mathrm{tu}}$, and disk temperature $T$; the latter two are assumed to be constant throughout the disk. Geometrical parameters are from [2] and [3].

Our preliminary results for $U$ Gem show agreement between observation and theory for the line intensities and line ratios, indicating that both $\mathrm{Ca}$ II and He I emission lines can show up in an isothermal AD. However, the line profile shapes are not matched correctly by the simple model disk, giving rise to a surface density and/or a temperature gradient.

Two models are shown for $T$ Leo demonstrating the sensitivity of model spectra on temperature.

Acknowledgement. WH thanks the Belgian Federal Scientific Services (DWTC/SSTC), project number $\mathrm{SC} / 005$, for funding.

\section{References}

1. Hummel, W., 1994, A\&A, 289, 458

2. Marsh, T.R., Horne, K., Schlegel, E.M., et al., 1990, Ap. J., 364, 637

3. Shafter, A.W., Szkody, P., 1984, Ap. J., 276, 305 



Figure 1. Calculated emission lines of the model disk compared with the continuum subtracted spectrum of $U$ Gem in quiescence.
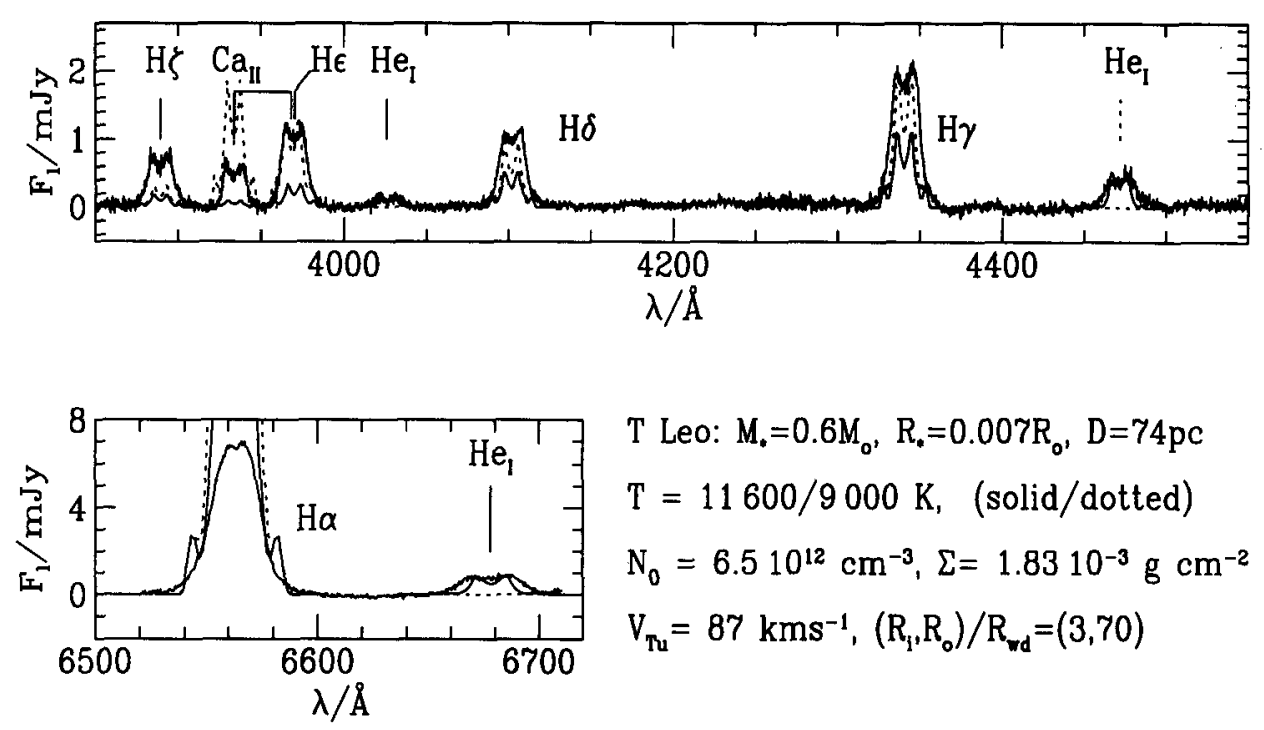

Figure 2. Calculated emission lines of two model disks with $T=11600 \mathrm{~K}$ (solid) and $T=9000 \mathrm{~K}$ (dotted) compared with the continuum subtracted spectrum of $\mathrm{T}$ Leo in quiescence. 